criteria which included, apart from astronomical considerations, questions like availability of water, nearness to a reasonably large city, etc. In view of the magnitude of the VLT investment, it is not clear that these last criteria should have the same weight today and a new search has been made to see if substantially better sites exist, without excluding from consideration ease of operation, infrastructure needs, or the personal comfort of the staff.
Studies have been mainly concentrated on a coastal region of Northern Chile, which seems to offer an outstanding climate, but space on the available summits is limited. Cerro Paranal located about $150 \mathrm{~km}$ south of Antofagasta, a few kilometres from the coast, and at 2650 m elevation - has been monitored for more than three years and appears an excellent candidate. On the basis of presently available data, Paranal appears to be supe- rior to any known site in the world for cloudless nights and is also extremely dry. It is a serious candidate for the VLT.

\section{Schedule}

The present plans foresee that the first telescope could be ready in 1995 and the project could be completed by the year 2000. An extensive instrumentation programme will be developed in collaboration with national astronomical institutes.

\title{
EPS Workshops
}

New achievements in physics not only satisfy our scientific curiosity, but can have often important technical and economic consequences. This new scheme of EPS Workshops strengthens the links between academic research and industrial development in Europe by bringing together competent representatives of both sides in special fields, where a broad collaboration is desired.

"Magneto-optical Recording" is such a characteristic field, where the creative hybridization between materials research and technical ingenuity is a decisive factor for economic success in a very competitive international market. The goal is to reach much faster, better and denser storage of information by replacing the usual combination of magnetic layers and inductive heads by modern optical devices, such as lasers, interacting with magneto-optically active magnetic materials.

Light is refracted and absorbed by matter through its interaction with the electrons present. In ferromagnetized materials, the magnetic electrons spin and rotate around parallel axes so that the optical properties are different for light waves of different polarity; a ferromagnet brought between two crossed polarizers may look dark or bright depending on the angle of its magnetization with respect to the light beam. This effect can be used for reading by a polarized laser beam the binary information stored in a magnetic tape or disk much faster and more conveniently than by small induction coils which usually need to fly at tremendous speed for very small distances over the surface. The advantages in terms of density, precision and quality of using magneto-optical techniques for recording are evident to anyone who has listened to a modern compact disk player.
The industrial realisation however of such magneto-optical devices requires the development of not only advanced optical and electronic apparatus, but also new magnetic materials, which combine the physical properties desired with a large magneto-optical activity. For this task one needs much more fundamental knowledge about the chemistry, electronic structure, magnetism and optics of magnetic alloys and compounds than can be found in the present literature.

"SQUID" are in a rather different economic category at the present time, but to exploit the potential of these low temperature quantum devices a similar meld of science and different technologies is needed and the close cooperation between academie and industry.

SQUID is an acronym for Superconducting Quantum Interference Device. Its working principle is based on flux quantization and the Josephson effect. Practically, SQUIDs are very sensitive magnetometers, working at a temperature as low as that required by the super- conducting materials of which they are made. Using an inductive coupled coil, such devices can also be used as low impedance, high sensitivity ammeters. There are basically two kinds, the RF SQUID and the DC SQUID, but even if their working principles are very different, their behaviour from the point of view of the user is much the same.

For frequencies lower than about 100 $\mathrm{MHz}$ there is no other amplifier with such a high sensitivity to energy changes. In fact their sensitivity, i.e. the minimum magnetic energy detectable for unit bandwidth, is usually expressed in units of the Planck constant. Often such a device is near to the limit imposed for an amplifier by quantum mechanics and is one of the few that explicitly makes use of quantum mechanics.

In many fundamental physics experiments the SQUID is the only device that can give the sensitivity required. On the other hand, the need for low temperature and special low noise, linearizing electronics excludes its use in many applications.

\section{The Future of Magneto-Optical Recording}

\section{Bad Honnef, 2-4 November 1987}

\section{Themes}

The workshop was devoted to discussions of basic research in magnetooptics and research and development on magneto-optical recording in Europe. Some 50 people from 11 countries participated, half of whom were from industrial R \& D laboratories. Of the 13 papers presented again half were from industrial $R$ \& $D$ laboratories and half from governmental and university research laboratories.
Without going into the detail of the special presentations* we summarize here the final discussion which reflects the essential goal of the workshop: To assess the status of magneto-optical recording in Europe, compared with that in Japan and the USA.

\footnotetext{
- A limited number of copies of the presentations are available from the EPS Secretariat, price SFR 50.-.
} 